\title{
Immune Checkpoint Inhibitor Therapy: A Boon to Elderly Patients with Advanced Melanoma?
}

\author{
Tsuyoshi Muta
}

Key words: melanoma, immune checkpoint inhibitor, elderly patient

(Intern Med 57: 2287-2288, 2018)

(DOI: 10.2169/internalmedicine.0686-17)

In this issue of the journal Internal Medicine, Mashima and colleagues present a very impressive image of a case of malignant melanoma that spread in flat on the left arm (1). At a glance, the patient's forearm was diffusely colored in black pigmentation that appeared exactly like a tattoo (1). Superficial spreading melanoma is the major type of melanoma. In contrast, such cases with a diffuse, progressive appearance are thought to be quite rare. According to a nationwide registry analysis that was conducted in Japan between 2011 and 2013, the crude incidence of invasive cutaneous melanoma per 100,000 person-years was 1.24 (2). Although less frequent in comparison to Western countries, cutaneous involvement is common among all types of melanoma, and a quarter of the cutaneous melanomas were located in the upper limb. General practitioners should consider the possibility of melanoma in the differential diagnosis of unusual skin pigmentations.

With regard to treatment of advanced melanoma, cytotoxic chemotherapy showed limited efficacy; the overall response rate (ORR) was approximately $10 \%$, the median progression-free survival (PFS) was approximately two months, and the median overall survival (OS) was less than ten months (3). The treatment of melanoma has dramatically changed through the development of targeted therapy and immunotherapy. Approximately half of melanomas contain a mutation in the gene that encodes BRAF, mutations of which are associated with high-risk features, primary truncal development, younger age, lack of chronic skin damage, and a worse prognosis (4). BRAF inhibitor treatment has been shown to be effective. The median PFS for BRAF inhibitor treatment was reported to be 9.4 months, while the median OS was 25 months (5). The most common adverse event reported in association with this treatment was pyrexia, while the most common grade 3-4 adverse events included hyponatremia and neutropenia (5).

Monoclonal antibodies that inhibit programmed cell death
1 (PD-1) or its ligand (PD-L1) have recently been developed. When PD-1 on T cells attaches to PD-L1 on the surface of cancer cells, the $\mathrm{T}$ cells become inactive. The inhibition of PD-1/PD-L1 interaction can activate the $\mathrm{T}$ cells to attack cancer cells. The administration of nivolumab, an antiPD-1 antibody, to previously untreated melanoma patients without BRAF mutations achieved an ORR of $40.0 \%$, a median PFS of 5.1 months, and a 1-year OS rate of $72.9 \%$ (6). The administration of pembrolizumab, another anti-PD-1 antibody, to previously untreated or treated melanoma patients achieved a median OS of 31 months (7). The administration of ipilimumab, an inhibitor of cytotoxic T-lymphocyteassociated antigen 4 (CTLA-4), can also augment antitumor immunity, and showed a durable response in some patients; the plateau in the OS rate curve beyond three years ranged from $20 \%$ to $26 \%$ (8).

Immunotherapy is free from direct myelosuppression and direct organ toxicities; thus, it is expected to be useful for the treatment of elderly patients. Actually, the oldest patient in the phase 3 study of anti-PD-1 was 86 years of age (6). However, immune-related adverse events (i.e., interstitial pneumonia, colitis, hypothyroidism, liver dysfunction, skin rash, type 1 diabetes, myasthenia gravis, neuropathy, and myositis) are disadvantageous (6-8). In patients of $\geq 80$ years of age, the efficacy and toxicity of immunotherapy against metastatic melanoma were comparable to the efficacy and toxicity in younger patients (9). When patients of $<65$ years of age were compared to those of $\geq 70$ years of age, the incidence of grade 3-5 adverse events was $58.4 \%$ vs. $71.7 \%$, respectively, while the incidence of events leading to discontinuation was $14.4 \%$ vs. $19.8 \%$, (10). These results suggest the possibility that elderly patients may have an increased rate of adverse events. Naturally, a careful evaluation of general conditions and a geriatric assessment are necessary for the proper management of immune checkpoint inhibitor treatment and immune-related adverse events. 
Actually, there were no data on the BRAF mutation status of the presented patient (1). Cytotoxic agents seemed to be too toxic for such an elderly patient; thus, the immunotherapy was the only treatment option. Immunotherapy is reported to show superior efficacy in patients with low LDH levels and with metastasis to the lymph nodes, soft tissue, and/or lung (11). The presented patient seemed to have a high tumor burden and liver metastasis (1); thus, the limited efficacy of anti-PD-L1 couldn't be helped. Specific trials should be performed to verify whether immunotherapy can improve the survival outcomes, including the quality of life, in elderly patients with advanced melanoma.

\section{The author states that he has no Conflict of Interest (COI).}

\section{References}

1. Mashima E, Sawada Y, Yoshioka M, Nakamura M. Tattoo-like spread malignant melanoma. Intern Med 57: 2441, 2018.

2. Tomizuka T, Namikawa K, Higashi T. Characteristics of melanoma in Japan: a nationwide registry analysis 2011-2013. Melanoma Res 27: 492-497, 2017.

3. Patel PM, Suciu S, Mortier L, et al. Extended schedule, escalated dose temozolomide versus dacarbazine in stage IV melanoma. Eur J Cancer 47: 1476-1483, 2011.

4. Long GV, Menzies AM, Nagrial AM, et al. Prognostic and clinico- pathologic associations of oncogenic BRAF in metastatic melanoma. J Clin Oncol 29: 1239-1246, 2011.

5. Long GV, Weber JS, Infante JR, et al. Overall survival and durable responses in patients with BRAF V600-mutant metastatic melanoma receiving dabrafenib combined with trametinib. J Clin Oncol 34: 871-878, 2016.

6. Robert C, Long GV, Brady B, et al. Nivolumab in previously untreated melanoma without BRAF mutation. N Engl J Med 372: 320-330, 2015.

7. Ribas A, Hamid O, Daud A, et al. Association of pembrolizumab with tumor response and survival among patients with advanced melanoma. JAMA 315: 1600-1609, 2016.

8. Schadendorf D, Hodi FS, Robert C, et al. Pooled analysis of longterm survival data from phase II and phase III trials of ipilimumab in unresectable or metastatic melanoma. J Clin Oncol 33: 18891894, 2015.

9. Friedman CF, Horvat TZ, Minehart J, et al. Efficacy and safety of checkpoint blockade for treatment of advanced melanoma in patients age 80 and older. J Clin Oncol 34 (suppl): 10009, 2016.

10. Singh H, Kim G, Maher VE, et al. FDA subset analysis of the safety of nivolumab in elderly patients with advanced cancers. J Clin Oncol 34 (suppl): 10010, 2016.

11. Weide B, Martens A, Hassel JC, et al. Baseline biomarkers for outcome of melanoma patients treated with pembrolizumab. Clin Cancer Res 22: 5487-5496, 2016.

The Internal Medicine is an Open Access article distributed under the Creative Commons Attribution-NonCommercial-NoDerivatives 4.0 International License. To view the details of this license, please visit (https://creativecommons.org/licenses/ by-nc-nd/4.0/).

(C) 2018 The Japanese Society of Internal Medicine Intern Med 57: 2287-2288, 2018 